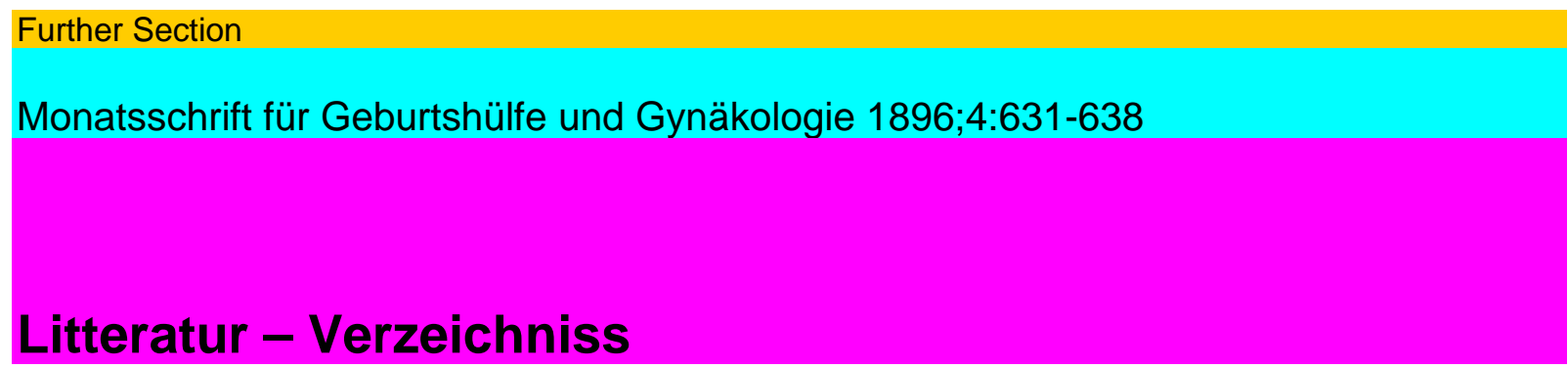

\title{
I. Geburtshilfe.
}

1. Bücher. Monograjphien. Berichte.

Eendiconto clinico del anno scolastico 1895-96 (dal 10. November 1895 al 31. Luglio 1896) dell'Istituto di clinica ostetricia e ginecologica della regia università di Genova, directo dal Prof. Luigi Acconci. Rivista d Ostetr., Ginec. e Pediatria. Nov. 1896.

Kossmann, R., Die geburtshilfliche Praxis in kurzer Darstellung. Med. Biblio-thek f. prakt. Aerzte. 85-89. Leipzig. C. G. Naumann.

-Veit, J., Die Ziele des geburtshülflich-gynäkologischen Unterrichts. Rede. Leiden 1896.

2. Allgemeines.

Ahlfeld, P., Die Bedeutung der äusseren Untersuchung der Gebärenden gegenüber der inneren. (Aus der Universitäts - Frauenklinik in Marburg.)

Deutsche med. Woch. 1896. No. 44. Arenhövel, W., Ein interessantes Drillingsei. Ein Beitrag zur Frage der Naeh-

empfängniss. Diss. Halle 1896. Dührssen, A., Ueber chirurgische Fortschritte in der

Geburtshilfe. Deutsche

med. Woch. 1896. No. 45. Hallervorden, E., Der Zusammenhang chemischer und nervöser Vorgänge im

Wochenbett. Diss. Würzb. 1896. ínzani, L’ostetricia moderna ed il suo indirizzo. Ann. di Ost. e Gin.

1896. No. 9. Rissmann, Zur Schwangerschaftsdiagnose in den ersten Monaten. Centralbl. f. Gyn. 1896. No. 43. Schober, Pariser Brief. Deutsche med. Woch. 1896. No. 43. Vahle, Ueber das Vorkommen von Streptokokken in der Scheide Gebärender.

Zeitschr. f. Geb. u. Gyn. XXXV. 2.

3. Physiologie und Pathologie der Schwangerschaft, der Geburt-und des Wochenbet tes. Ahlfeld, F., Zur Diagnose der Zwillingsschwangerschaft. Zeitschr. f. Geb. und Gyn. XXXV. 2. Becking, A.G. Th., Jcterus gravidarum. Bijdrag $\theta$ tot de pathologie der zwanger-schap. Nederl. Tijdschr. v. Verlosk. en Gyn. 1896. Afl. 4.

Cameron, Murdoch, Retroversion of the Pregnant Uterus. Reducible and Irreducible, with a New Method of Treatment. Brit. Med. Journ. 1896. No. 1870.

Eklund, Abraham Fredrik, Albuminuria in Pregnant and Puerperal Women. Amer. Journ. of Obst. Oct. 1896.

Grunow, W.. Herzerkrankungen in der Schwangerschaft mit besonderer Berück-sichtigung der Klappenfehler und ihrer Therapie. Diss. Greifswald 1896.

632 Litteratur -Verzeichnis.

Hart, D. Berry, The Nature and Diagnosis of the So-called Fleshy Mole. Brit.

Med. Journ. 1896. No. 1869. Heller, E., Ueber die Nierenerkrankungen Schwangerer. Diss.

Berlin 1896, Herff, Otto v., Beiträge zur Lehre von der Placenta und von den mütterlichen 
Eihüllen. Zeitschr. f. Geb. u. Gyn. XXXV. 2. Jarvis, John, An Uncommon Midwifery Case. Brit. Med. Journ. 1896. No. 1871. Kerron, R. G. Mc, Antistreptococeus Serum in Puerperal Fever. Brit. Med.

Journ. 1896. No. 1867. Kroner, M., Ueber Chorea gravidarum. Diss. Berlin 1896. Lauro, Contribuzione casistica al parto per la fronte e considerationi. Arch, di

Ost. e Gin. 1896. No. 8 u. 9. Lesse, W., Ein weiterer Fall von Luftembolie bei Placenta praevia. Zeitschr. f.

Geb. u. Gyn. XXXV. 2. Löbell, G. H., Das Verhalten der Nachgeburt bei 500 Geburten. Diss. Würz-

burg 1896. Neumann, A., Kann Uterusruptur durch plötzlichen Blasensprung bei Hydramnios zustande koramen? Freund, H. W, Erwiderung auf vorstehenden Aufsatz. Deusche Med. Woch. 1896. No. 43. Noble, George H., One Hundred and Sixty-six Cases of Cancer of the $1 / 8$ egnant Uterus occurring since 1886. Atlanta Med. a. Surg. Journ. July a. Aug.

1896. Mesinelli, Note batteriologiche su di un abortu per tifo addominale. Ann. dí Ost. e Gin. 1896. No. 9. Schmarsoiv, K., Zwei Fälle ausgetragener Gravidität bei fibromatös degenerirtem

Uterus. Diss. Königsberg 1896. Schilling, H., Beitrag zur Kenntnis der hämorrhagischen Läsionen der Placenta.

Diss. Basel 1896. Spivak, C. D., Delivery at Term after Ten Previous Consecutive AbortionsAmer. Gyn. a. Obst. Journ. Oct. 1896. Succow, W., Ein Fall von centralem Dammriss mit Entwickelung des Kindes

durch denselben. Deutsche Med. Woch. 1896. No. 46. Thumim, Leopold, Casuistíscher Beitrag zur Complication der Schwangerschaft

mit Myomen. (Aus Prof. L. Landau's Frauenklinik in Berlin.) Wien.

Klin. Woch. 1896. No: 41. Tinley, W. E. F., Chorea Gravidarum: Induction of Labour: Death. Brit. Med.

Journ. 1896. No. 1868. Tuszkai, Zur Frage der Hyperemesis gravidarum. Ung. Med. Presse. 1. Nov. 1896. Westerode, A., Die Nierenaffectionen in der Gravidität und ihre Beziehungen zur chronischen Nephritis. Diss. Würzburg 1896. Wetterçreen. Carl, Oin hämatoma vulvae et vaginae post partum. Hygiea. 1896.

No. 9. Wilkinson, A. D., Phlegmasia Dolens. Amer. Gyn. a. Obst. Journ. Oct. 1896. Williams, D. John, The Value of Antistreptococcic Serum in the Treatment of

Severe Puerperal Septicaemia. Brit. Med. Journ. 1896. No. 1870.

4. Enges Becken. Bland-Sutton, J., Clinical Remarks on Some Interesting Pelvic Cases. Brit. Med. Journ. 1896. No. 1870. Uhl, F., Beitrag zur Beckenmes¹/8ung nach Skutseh. Diss. Jena 1896.

5. Geburtshilfliche Operationen und Therapie, Abortus,

Operationen im Wochenbett.

Ahlfeld, Perforation, Kaiserschnitt, künstliche Frühgeburt, spontane Geburt?

Zeitsch. f. prakt. Aerzte 1896. No. 21. Ahlfeld, F., Giebt Tympania Uteri eine Indikation zur Entfernung des Uterus

in partu? Zeitschr. f. Geb. u. Gyn. XXXV. 2. Baker, Oswald, Champetier de Ribes's Bag in Placenta Praevia. Brit. Med.

Journ. 1896. No. 1867. JBracht, H.. Beitrag zur Statistik der sectio caesarea unter besonderer Berück-

sichtigung der durch Beckengeschwülste bedingten Indikationen. Diss. 
Kiel 1895.

Litteratur - Verzeichnis.

633

Brothers, A , Cäsarean Section. Amer. Journ. of Obst. Oct. 1896.

Bùttner, R., Beitrag zur Beurteilung des therapeutischen Wertes des Curette-

ments bei Metrorrhagien nach Abort oder Geburt. Diss. Kiel 1896. Byers, W. John, A Plea for the Eearly Recognition and Treatment of Puerperac

Fever. Brit. Med. Journ. 1896. No. 1869. McCaw, John, Notes of a Case of Premature Labour presenting some Unusual

Features. Lancet. 1896. No. 3818. Cholmogoroff, S. S·, Die Einführung von physiolvgischen Kocbsalzlösung in den

Órganismus bei grossen Blutverlusten während und nach der Geburt. Diss.

Leipzig 1896. Deïbert, J., Ein Beitrag zur Symphyseotomie-Frage. Diss. Giessen 1896.

Fothergill, W. E., Walcher's Position in Parturition. Brit. Med. Journ. 1896.

No. 1870. Herrenschneider, Beitrag zur causalen Behandlung des Kindbettfiebers. Diss.

Strassb. 1896. Longyear, H. W., The Treatment of Puerperae Infection. Amer. Journ. of Obst. Oct. 1896. Neumann, Emil, (Livland). Ueber prophylaktische Dammincisionen beim Durchtritt des Kopfes. Wien. med. B1. 1896. No. 44. Purslow, 0. B., On Perforation of the Aftercoming Head. Brit. med. Journ,

1896. No. 1870. Schaeffer, Bekämpfung der post partum- und parenchymatösen HöhlenBlutungen

mittelst nicht drainirender, imprägnirter Gaze. Munch, med. Woe.. 1896.

No. 40. . Schmidt, H., Ueber die Anwendbarkeit der Verkleinerung des nachfolgenden

Kopfes durch den Wirbelkanal bei räumlichem Missverhältnis (Cohnstein-

sches Verfahren). Diss. Kiel 1896. Stanmore, E., Bishop., Post partum Haemorrhage and its

Treatment. Lancet.

1896. No. 3818. Voiges, W., Beitrag zur Statistik, Prognose und zu den Indicationen der

Symphy-

siotomie. Diss. Kiel 1896.

6. Ektopische Schwangerschaft.

Blumenthal, W., Ueber Graviditas tubaria. Diss. Greifswald 1896.

Henrotin, Fernand, Early Rupture in Extra-uterine Pregnancy and its Treatment.

Amer. Journ. of Med. Sciences. Nov. 1896. Sehulze-Vestinghausen, A., Ueber die Laparotomie wegen freier intraperitonealer

Blutung in Folge von früher Tubenschwangerschaft. Diss. Kiel 1895. Weil, J., Spontanruptur des

graviden Homes bei Uterus bicornis im 9. Schwanger-

schafts-Monate mit tödlichem Ausgang. Centralbl. f. Gyn. 1896. No. 46.

7. Eclampsie.

Berger, J., Ein Fall von Eklampsia graviditatis. Diss. Bonn 1896.

Catto, William M., Puerperal Eclampsia. Amer. Gyn. a. Obst. Journ. Oct. 1896.

Đrejer, Om den obstetriciske Behandling af Puerperaleklampsien. (Ueber die

gebh. Behandlung der Puerperaleklampsie) Norsk. Mag. f. Laegev

1896. No. 9. Gibson, John R., A Case of Puerperal Eclampsia without Renal Symptoms;

Blood-letting; Recovery. Lancet. 1896. No. 3817. Latimer, John W., A Case of Diphtheria, Ushered in by Eclampsia-Complicaced 
by Labor at Term. Annals of Gyn. a. Paed. Oct. 1896. Mangiagalli, Sul trattamento dell' eclampsia. Amer. di Ost. e. Gin. 1896. No. 9.

8. Osteomalacie. Breidthardt, Br., Zwei Fälle von Castration bei Osteomalacie. Diss. Kiel. 1896.

9. Foetus, Neugeborenes, Lactation.

Bergkammer, Fr., Ueber einen Fall von Teratom, rudimentärem Parasiten (En-gastricus) oder Inclusio foetalis abdominalis des Beckens bei einem elf-monatlichen Knaben. (Aus dem Fr. Krupp'schen Krankenhaus in Essen.-Deutsche Med. Woch. 1896, No. 44.

634 Litteratur -Verzeichnis.

Bosse, E., Zur Casuistik der Atresia ani. Diss. Greifswald 1896.

Clark, Alfred, A Case of Absence to the Thymus Gland in an Infant. Lancet

1896, No. 3816. Coulthard, W. J. C, An Acephalons Infant. Lancet 1896, No. 3816. Dellevie, Heinrich, Zur Frage der künstlichen Emähruug der Säuglinge. Centralbl.

f. Kinderheilk. 1896, H. 6. Feige, E., Ueber die Todesursachen der Säuglinge bis zum 6.

Lebensmonate

incl. nach den Protokollen der Jahre 1886-95. Diss. Kiel 1896. Fischl und v. Wuntichheim,

Schutzkörper im Blute des Neugeborenen. Prager

Zeitschr. f. Heilk. Bd. XV. Hermary, Des hemorrhagies gastro - intestinales chez le nouveau-né.

These.

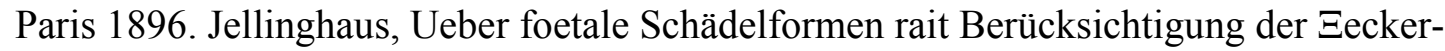

schen Aetiologie der Gesichtslagen. Diss. Berl. 1896. Kautzsch, W., Zur Obliteration der fötalen Gefässe. Diss. Halle 1896. Knoepfelmacher, Wilhelm, Das Verhalten der roten Blutkörperchen beim Neugeborenen mit K, ücksicht auf den Icterus neonatorum. (Aus der Xlinik

des Herrn Prof. Schauta in Wien.) Wien. klin. Woch. 1896, No. 43. Menke, A., Ein Beitrag zur

Statistik und Prognose der Geburtsverletzungen

des Kindes. Diss. Kiel 1895. Meyer, Hans, Wiederbelebung asphyktischer Neugeborener.

Corresp.-B1. f.

Schw. Aerzte. 1896. No. 21. Schivoboda, Norbert, Zur Aetiologie der Melaena. (Aus der niederösterreichi-

schen Landesfindelanstalt.) Wien. klin. Woch. 1896, No. 41. Turner, G. A., The Successful Preventive Treatment of the Scourge of St. Kilda.

(Tetanus Neonatorum.) Brit. Med. Journ. 1896, No. 1869. Twombly, Edward, L., A Case of Anencephalia. Ann. of Gyn. a. Paed. Oct. 1896. Wendeler, P., Bemerkungen zudemAufsatz des Herrn Geh.-Rat B. S. Schultze: „Ueber die beim Scheinrod Neugeborener vorliegenden Indikationen" in No. 37

d. Bl. Centralbl. f. Gyn. 1896. No. 45.

II. Gynäkologie.

10. Bücher. Monographien. Berichte.

Fliess, W., Die Beziehungen zwischen Nase und weiblichen Geschlechtsorganen.

In ihrer biologischen Bedeutung dargestellt. Leipzig u. Wien 1897. F.

Deuticke. Menge u. König, Bacteriologie des weiblichen Genitalkanales. Leipzig 1896.

Ed. Resold (Arthur Georgi). $₫$ Seml , Oscar, Om de papillaere Ovarial-Kystomer.

(Monographie.) Beilage zum

„Norsk Magazin for Laegevidenskaben”, Okt. 1896. Verhandlungen der Gesellschaft für

Geburtshülfe und Gynäkologie zu Berlin

vom 12. bis 26. Juni 1896:

Waldeyer, Die Lendenraute und die Kreuzraute. (Vortrag.) 
Derselbe, Die Lage des Eierstocks. (Vortrag.)

Mackenroät, Blasensteine um Silkwormfäden.'

Derselbe, Uterus Didelphys.

Kiefer, Meningococcus und Gonococcus.

Kossmann, Formalincatgut.'

Hollander, Kolpoplastik.

Winter, Zur Pathologie des Prolapses. (Vortrag.) Zeitschr. f. Geb.

u. Gyn. XXXV. 2. Wilheim, J., Die Nervosität der Frauen. 2. Aufl. Wien 1896. Windscheid,

Franz, Neuropathologie und Gynäkologie. (Eine kritische Zu-

sammenstellung ihrer physiologischen und pathologischen Beziehungen.)

Berlin 1897. S. Karger. Wolf, M., Die physische und sittliche Entartung des modemen Weibes.

4. Aufl.

Dresden 1896.

11. Allgemeines.

Bannermann, G. Gilbert, Naevus Pigmentosus. Brit. Med. Journ. 1896. No. 1870.

Bodon, K., Ueber drei mit Ovariinum siccum (Merck) behaudelte Fälle, dar-

unter ein Fall von Epilepsie. Deutsche med. Wochenschr. 1896. No. 44.

Litteratur -Verzeiclinis. $\quad 635$

Bossi, A. proposito dei rapporti fra ovulazione e raenstruarione. Ann. di Ost.

$\theta$ Gin. 1896, No. 9. Braithwaite, James, Case Illustrating the Use of Transfusion and of Ozygeu

Inhalation in Certain Circumstances following Abdominal Section. (Leeds

General Infirmary) Brit. med. Journ. 1896. No. 1867. Chrobak, R., Ueber den Unterricht in der

Frauenheilkunde. S. A. aus „Berichte

aus der Klinik." Wien 1896. In Kommission bei Alfred Holder. 111, Edward J., Nine Mouths of Abdominal and Gynecolegical Surgery. Amer.

Journ. of Obst. Okt. 1896. Kast, Ueber den Werth der arzneilichen Antipyretica. Verh. d.

Congresses $\mathrm{f}$.

inn. Med. Wiesbaden 1896. J. F. Bergmann. Newman, Henry Parker, Woman and her Diseases vs Gynecology. Amer. Gyn.

a. Obst. Journ. Okt. 1896. Beamy, Thaddeus A., In Memoriam: Robert Battey. Amer. Gyn. a.

Obst. ' Journ. Oktober 1896.

12. Anatomie und pathologische Anatomie. Entwickelungs-geschichte. Missbildungen.

Blacker, G. F., A Case of True Unilaterael Hermaphroditism with Ovetestis

occurring in Man, with a Summary and Criticism of the Recorded Cases

of True Hermaphroditism. Trans, of the Obst. Soc. of London XXXVIII. 3. McCann, Frederick

John Some Abnormalities of the Female Generative Organs.

Amer. Journ. of Med. Sciences. Oct. 1896. v. Dittel, Leopold jun., Ueber die elastischen Fasern der Gebärmutter. Wien.

Klin. Rundschau. 1896. No. 26 und 27. Edge, Fred., A Case of Uterus Bicornis Septus. Brit.

Med. Journ, 1896. No. 1870. Epstein, Em., Persistirender Urachus. Ung. med. Presse 1. Nov.

1896. van Meerdervoort, N. J. F. Pompe, Het ovarium gedurende de menstruatie. De

vorming van het corpus luteum. Nederl. Tijdschr. v. Verlosk. en Gyn.

1896. Afl. 4. Remfry, Microscopical Sections of Uterus showing Chorionic Villi and Doubtful

Sarcoma. Trans, of the Obst. Soc. of London. XXXVIII. 3. Wanser, A., Ueber Missbildungen bei Hydramnion. Diss. Tubingen 1896.

13. Vulva. Scheide, Damm. Beckenbindegewebe. 
Braun', 1·L, Ueber die traubenförmigen Sarkome der Vagina und des Uterus.

Diss Greifswald 1896. Brothers, A., Reports of Some Interesting Cases: Impacted Pessary Vaginal

Section with Hemarrhage. Amer. Journ. of Abst. Oct. 1896. Lanqe, G., Ueber einen Fall von primärem, multiplen Vaginalkarcinom. Diss.

Kiel 1896. Phillips, John, Anterior Colpotomy. Trans, of the Obst, Soc of London XXXVIII. H. 3. Rode, Emil, Sterilítas hymenalis vulvalisque, non gonorrhoica. Tidsskr. f. d.

norske Lægeforening. 1896. No. 19.

14. Uterus und Operationen an demselben.

Bee, Le, Total Hysterectomy for Large Fibroids: New Methods. Brit. Med.

Journ. 1896. No. 1870. Cogrel, Hystéroscope. Son instrumentation. Son manuel opératoire. Ses

re-

sultats aequis. These. Paris 1896. Coléris, Traitement des rétrodéviations utérines. Ann. de Gyn. et d'Obst.

Oct. 1896. Donald, Archibald, Intraperitoneal Hysterectomy and Hysterectomy by the Combined Method for Fibroid Tumours of the Uterus, with Notes of Cases.

Brit, Med. Journ. 1896. No. 1869. Dunn, B., Sherwood, Vaginal Hysterectomy by the Clamp Method. Amer. Journ.

of Obst. Oct. 1896. Helme, Arthur, The Technique of the Vaginal Total Extirpation, with Notes of

Ten Cases. Brit. Med. Journ. 1896. No. 1870.

636 Litteratur -Verzeichnis.

Hirschmann, Ueber vaginale Totalextirpation des carcinomatösen Uterus. Diss.

Würzb. 1895. Hofmeier, M.. Ueber die Beziehungen zwiscben Myom und Sterilität. Berl.

klin. Ẃochenschr. 1896, No. 43. Lange, M., Ueber die Diagnose und Tlierapie des Carcinoma uteri. Smmlg.

zwangl. Abh. aus dem Geb. d. Frauenheilk. u. Geburtsh. Bd. I. H. 6.

Halle 1896. Karl Marliold. Derselbe, Ueber die Diagnose und Therapie des Carcinoma uteri.

Diss. Halle 1896. Lawrie, Macperson, Total Exstirpation of the Uterus, with Six Cases. Brit.

Med. Journ. 1896, No. 1870. Leech, Joseph William, Complete Absence of Vagina and Uterus.

Brit. Med.

Journ. 1896. No. 1867. Kühn, A., Ein Pall von Spindelzellensarkom im Corpus Uteri mit

cystischer

Degeneration. Diss. Greifswald 18S'6. Küstner, Otto, Ueber Indicationen und

Kontraindikationen der Myomotomie.

Smmlg. klin. Vortr. No. 164. Leipzig 1896. Breitkopf u. Härtel. Martin, Christopher, On

Haematometra and Pyometra. Brit. Med. Journ. 1896.

No. 1870. Inglis-Parsons, J., A Case of Malignaut Adenoma of the Uterus. Brit. Med.

Journ. 1896. No. 1870. Praetorius, E., Ein Pall von Adenoma malignum des unteren

Gebärmutter-

abschnitts. Diss. Halle 1996. Pompe von Meerdervoort, N. J. F·, Hypertrophia mucosae uteri.

Nederl. Trjdschr.

v. Verlosk. en Gyn. 1896. Afl. 4. Bichter, P., Partielle durch Tumoren bedingte

Uterusinversionen. Diss.

Berlin 1896. Bishntiller, John, H., The Employment of Gauze in the Uterine Cavity. Amer. 
Gyn. a. Obst. Journ. Oct. 1896. Boss, E., Fairfax, Note on the Technique of Ventriflxation of the Uterus. Brit.

Med. Journ. 1896. No. 1867. Wallace, John. Notes on the Evolution of Treatment of Uterine

Fibroids in the

Thornton Ward, Liverpool Royal Infirmary. Brit. Med. Journ. 1896.

No. 1870 White, Herbert, W., Practical Observations on the Electrical Treatment of

Uterine Mammary, and other Diseases. Brit. Med. Journ. 1896, No. 1869. Williams, Boger, The

Clinical Features of Uterine Caner. Lancet 1896.

No. 3816.

15. Ovarium. Tuben. Ligament e.

Braetz, A., Ein Fall von Endotheliom der Portio vaginalis. Diss. Halle 1896. Clarke, P. Augustus, The Relation of Malignant Disease of the Adnexa to the

Primary Invasion of the Uterus. Amer. Journ. of Obst. Oct. 1896. Cumston, Charles Greene, Torsion of the Pedicle in Ovarian Tumors. Ann. of

Gyn. a. Pael. Oct. 1896. Doran, Alban, Cases of Fibroma of the Ovary and Ovarian Ligament

removed

by Operation, with a Series of Afterhistories of Cases reported in the

r Transactions" since 1879. Trans, of the Obst. Soc. of London XXXVПI, H.3. Edebohls, George M., Shortening the Round Ligaments; Indications, Technique,

and Results. Ann. of Gyn. a. Paed. Oct. 1896. Gilliam, David Tod, Oopharectomy for the

Insanity and Epilepsy of the Female.

A Plea for Its More General Adoption. Amer. Journ. of Obst. Oct. 1896. Morse, H. Thomas,

Case of Dermoid Ovarian Cyst impacted in the Pelvis which

was removed by Abdominal Section during the Ninth Month of Pregnancy.

Trans, of the Obst. Soc. of London XXXVIII. 3. Nairne, J. Stuart, The Diagnosis of Early

Ovarian and Tubal Disease. Brit.

med. Journ. 1896. No. 1870. Williams, W. Roger, A Large Dermoid Ovarian Cyst Successfully

Removed by

Laparotomy. Brit. Med. Journ. 1896. No. 1867. BosinsM, B., Zur Lehre von den endothelialen Ovarialgeschwülsten, Zeitschr. f.

Geb. u. Gyn. XXXV. 2. South, Amand, Hydrosalpinx and Small Ovarian Cyst removed by

Anterior Colpo-

tomy. Trans, of the Obst. Soc. of London XXXVחI. H. 3.

Litteratur-Verzeichnis. 637

Schramm, J., Ein neuer Fall von Haematoma ligamenti rotundi uteri. Centralbl.

f. Gyn. 1896. No. 45. Thorn, W., Haematosalpinx profluens. Gont,ralbl. f. Gyn. 1896. No. 43.

Winter, A., Ergebnisse der in der Giessener Frauenklinik von 1888-1896 aus-

geführten Ovario- und Parovariotomieen. Diss. Giessen 1896.

16. Bauehhöhle. Bauchschnitt. Scheiden-Bauchschnitt.

Askanazy, M., Kann Darminhalt in der mens < hlichen Bauehhöhle einheílcn?

Virchow Archiv, Bd. 146, Heft 1. Deitermann, 01.. Ueber die Heilung der Perforativnspeiitonitis nach Laparotomie.

Diss. Wiirzburg 1896. Doyen, Eugene, Traitement des Suppurations pelviennes de

Thystérectomie ab-

dominale totale, traitement chirurgical des retro deviations utérínes, du

meilleur mode de fermeture de la paroi abdominale. Paris 1896. Institut 
international de bibliographie scientifique. Dunning, L. H., Shall Hysterectomy be Performed in Inflammatory Diseases of the Pelvic Organs. Amer. Journ. of Obst, Oct. 1896. Frank, Louis, Operative Procedures for Pelvic Inflammation. Amer. Gyn. a.

Obst. Journ. Oct. 1896. Graupner, I!., Ueber Hernien der linea alba. Diss. Jena 1896. Holmes, Bayard, Tubercular Peritonitis. Ann. of Gyn. a. Paed. Oct. 1896. Kehrer, F. A., Bauchnaht bei Laparotomie. Centralbl. f. Gyn. 1896. No. 43. Kossmann, R., Ueber die Verhütung der Bauchhernie. Centralbl. f. Gyn. 1896.

No. 43. Marjantschik, N. P., Ein Fall von Laparotomie an einer Neugeborenen wegen Hernia funiouli umbilicalis. Diss. Leipzig 1896. Sannemann, K.. Ueber die Beckenhochlage bei Operationen in der Bauehhöhle.

Diss. Kiel 1896. Steffeck, Zur vaginalen Coeliotornie. Beil. Klin. Woch. 1896. No. 44. 17. Harnorgane. Fisteln.

Baldy, J. M·, Surgical Injuries to the Ureter. Amer. Gyn. a. Obst. Journ.

Okt. 1896. Blumer, Georg, Notes on Two Cases of Ureteral Abnormality. Bull, of Johns Hopk. Hosp. Sept.-Okt. 1896. Cordier, A. H,, Moeable Kidney. Local and Remote Results. Amer. Journ. of

Obst. Okt, 1896. Graf, F., Urachusfiste'n und ihre Behandlung. Diss. Berlin 1896. Iüeinschmidt, E.. Ein Beitrag zur Casuistik der Fremdkörper der weibl. Blase u. die Methoden ihrer Entfernung. Diss. Würzb. 1896. Leech, Joseph William, Complete Inversion of the Bladder. Brit. med. Journ.

1896. No. 1868. Morgan, George, A Case of Dilated Urachus Accidentally Opened whilst Performing Abdominal Section for Peritonitis, Rocovery. Lancet 1896.

No. 3817. Tóth, S., Hydronephrose in Folge schíefer Einmündung des Ureters. Laparonephrektomie. Centralbl. f. Gyn. 1896. No. 43.

18. G o n o r r h o e.

Caun, J. Mc, The Aetiology of Gonorrhoea. Trans, of the Obst. Soc. of London. XXX VIII. 3.

19. Hysterie.

Moore, James E., Nymphomania Cured by Hysterectomy. Amer. Journ. of Obst.

Okt. 1896. Schmitt, W., Ueber einen auf chirurgischem Wege geheilten Fall von Paradoxia sexualis. Diss. Berlin 1896.

20. Mamma.

Arnoldt, W., Operative Erfolge bei Carcinoma mammae. Diss. Halle 1896.

Jlcnataschrift für Gebnrtshülfe und Gynäkologie. 1896. Bd. IV. Hett 6. 43

638 Litteratnr- -Verzeichnis.

Bach-, Oscar, Ueber das Fibro - Adenoma Mammae proliferum. Diss. Leipzig.

1896. Hellier, John B., On the Nipple Keflex. Brit, Med. Journ. 1896. No. 1871. Schroder, K., Mastitis in der Schwangerschaft. Diss. Marburg 1896. Snow, Herbert, Notes on Three Hundred Cases of Breast Excision for Malignant

Disease. Brit, Med. Journ. 1896. No. 1868.

21. Technisches. Akiurgie. Bishop, Ovariotomie-Troikar. Aerztl. Polytechn. 1886. No. 10.

Fleisdihauer E. Chirurgisclies Nälitmateria) zu Ligaturen und verlomen Nähten

mit besonderer Berücksichtigung der Seide. Diss. Erlangcn 1896. Friedlander, Georg, Badehalter für Säuglinge. Aerztl. Polytechn. 1896. No. 10. Hue, Otto, Eine neue Naht der Bauchdecken. Wien. Med. Presse 1896. No. 40. derselbe, Eine neue Unterbindungsnadel. Betz: 
„Memorabilien.” 189õ. H. 3. Lane, W. Arbuthnot, Improved Needle-holder. Lancet, 1896. No. 3815. Reineckf, Untersuchungs- und Massage-Sopha, gleiclizeitig verstellbar als Operations- und Untersuchungstiscli, resp. Untersuchungsstuhl. Munch. med. Woch. 1896. No. 40. Schaeffer, 0., Ueber die Verwertung der anhydrophilen Nähseide. Centralbl. f.

Gyn. 1896. No. 46. -Warhsmann, S., Heftklaminern für Wunden. Berl. Klin. Woch. 1896. No. 45 .

22. Narkose. Antiseptic a. iïvgelbrecht, .).. Die Narkosen der Bonner Fraucnklinik im Jahre 1895. Diss.

Bonn 1896. Langstein, J"ellotin als Schlafniittel. I'rag. med. Woch. 1896. No. 40. Zur Narkotiijróngs $1 / 8$ age, Aerztl. Polytechn. 1896. S. 155. Randolp1. líobcrr. L. Absolute Alcohol a >a Disinfectant for Instiui $>\cdot$ ents. A

Bacteriological Study. Bull, of Johns Hopk. Hosp. Sept.-Oct. 1890. Stobiva,-scr,

(lilvrofornimaske mit Sriinbinde. Deutsche med. Woch. 1896. No. 42. Jubilee, of Anaesthesia.

History of the Discovery.

.Squire, William, The First Operation under Ether in Great Britain.

Buxton, Dudley W., Fifty years of Anasthetics. Brit. Med. Journ.

Verantwortlicher Eedacteur: Prof. Dr. M. Sänger in Leipzig.

1896. No. 1868. 